SciDioc

\section{A Review On Medication-Related Osteonecrosis Of The Jaw: Definition and Best Practice For Prevention, Diagnosis, and Treatment}

\section{International Journal of Dentistry and Oral Science (IJDOS) ISSN: 2377-8075}

Research Article

Keerthana ${ }^{1}$, Lakshmi Thangavelu ${ }^{1 *}$

${ }^{1}$ Department of Pharmacology, Saveetha Dental College and Hospitals, Saveetha Institute of Medical and Technical Sciences, Saveetha University, Chennai, India.

\title{
Abstract
}

The aim is to review on medication related osteonecrosis of the jaw its definition, prevention, diagnosis and treatment. Skeletal complications due to osteoporosis or bone metastases are associated with considerable pain, increased mortality, and reduced quality of life. Agents that prevent bone resorption such as bisphosphonates or denosumab can reduce the risk of skeletalrelated events and are widely used in patients with osteoporosis or bone metastases. However, MRONJ can be treated and the likelihood of developing this condition can be reduced through prophylactic dental care and the maintenance of good oral hygiene. This review describes the incidence and pathophysiology of MRONJ and provide guidance for dental practitioners on the screening, prophylactic treatment, diagnosis, and management of patients with this condition.

Keywords: Osteonecrosis; Jaw; Resorption; Bisphosphonates; Denosaumab.

\section{Introduction}

Medication-related osteonecrosis of the jaw (MRONJ) is an uncommon condition that can occur after exposure to agents used to prevent bone complications, such as bisphosphonates or denosumab. In the majority of cases, MRONJ manifests as exposed bone in the maxillofacial region, although non-exposed MRONJ has also been recognised [1].

To reduce the risk of skeletal complications in patients with bone loss, resulting from long-term cancer treatment or osteoporosis, and in patients with malignant bone disease bisphosphonates and denosumab are predominately used [2] Bisphosphonates are small molecules that dock in hydroxyapatite-binding sites on bone surfaces. When osteoclasts begin to resorb bisphosphonate-impregnated bone, the liberated bisphosphonates bind to farnesyl pyrophosphate synthase inside the osteoclasts, ultimately leading to apoptosis [3]. MRONJ is more prevalent among patients who receives higher doses of bisphosphonates or denosumab than in patients who receive lower doses. The pathogenesis of
MRONJ is likely to be multifactorial and can involve a synergistic effect between local infection/trauma and decreased bone turnover after exposure to bisphosphonates or denosumab. Different mechanisms may be involved in the development of MRONJ in association with other agents. The importance of localised dental and periodontal infection in the development of MRONJ has been highlighted in recent animal experiments, which supported the findings from radiologic, histologic, microbiologic, and clinical studies [4]. A dental assessment is recommended, where feasible, before commencement of bisphosphonates, and any pending dental or oral health problems should be dealt with before starting treatment, if possible [5]. To explain the MRONJ disease frequency value, we have to consider two criteria: Therapeutic indications (osteoporosis/osteopenia and malignancy) and type of medication (BP and non-BP) AAOMS has proposed the following characteristics for MRONJ: treatment with antiresorptive or antiangiogenic medication currently or in the past, the bone remains exposed in the oral cavity for at least eight weeks with no healing, and the patient was not exposed to radiation therapy of the jaw in the past [6]. Intravenous (IV) BPs are widely used to treat bone metastases of malignant tumours such as multiple

*Corresponding Author:

Lakshmi Thangavelu,

Department of Pharmacology, Saveetha Dental College and Hospitals, Saveetha Institute of Medical and Technical Sciences, Saveetha University, Chennai, India. Email Id: Lakshmi@saveetha.com

Received: February 25, 2021

Accepted: March 04, 2021

Published: March 19, 2021

Citation: Keerthana, Lakshmi Thangavelu. A Review On Medication-Related Osteonecrosis Of The Jaw: Definition and Best Practice For Prevention, Diagnosis, and Treatment. Int J Dentistry Oral Sci. 2021;08(03):2036-2040. doi: http://dx.doi.org/10.19070/2377-8075-21000400

Copyright: Lakshmi Thangavelu ${ }^{\circ} 2021$. This is an open-access article distributed under the terms of the Creative Commons Attribution License, which permits unrestricted use, distribution and reproduction in any medium, provided the original author and source are credited. 
myeloma, breast cancer, prostatic cancer, as well as hypercalcemia of malignancy 4-7. Oral BPs are used to treat osteoporosis, osteopenia, Paget's disease, or osteogenesis imperfecta. The risk factors for the development of ONJ in oncology patients, in the order of importance, include: intravenous BPs, zoledronic acid, pamidronate, radiation thera- py, dental extraction, chemotherapy, periodontal disease, oral BP use, local suppuration, and denture use [7]. Demographic and systemic factors include: osteoporosis, glucocorticoid therapy, diabetes, erythropoietin therapy, tobacco use, hyperthyroidism, renal dialysis, and increasing age [8]. Significant risk factors for the development of $\mathrm{ONJ}$ in the osteoporotic population, in declining order of importance, include suppuration, BP use, dentalextraction, and anemia. Several hypotheses have tried to demonstrate the mechanisms of developing the disease. Given that the disease is multifactorial, it is unlikely that a single theory can explain its occurrence, and it is also unlikely that treatment is effective in all patients. The occurrence of numerous clinical and preclinical studies that reveal more evidence causes our hypotheses and treatment modalities to be in permanent change.

The first theory refers to bone remodelling inhibition. Antiresorptive drugs significantly decrease skeletal-related complications and relieve severe bone pain due to their direct effects on osteoclasts [9]. The primary mechanism of BPs and denosumab is to inhibit osteoclast function and increase apoptosis by different mechanisms, and this leads to altered bone remodelling, which is the leading hypothesis for ONJ development [10]. Another important theory refers to inflammation and infection, which have been considered an important component of ONJ. Adults' teeth are almost always extracted because they have periapical or periodontal inflammation, and it is well known that extraction is a highrisk factor for developing ONJ [11]. Studies identified bacteria, especialy Actinomyces species associated with active osteoclastic resorption on the necrotic bone surface. Angiogenes is inhibition is another major hypothesis at-tempting to explain the occurrence of ONJ. Bisphosphonates, especially nitrogen-containing BPs, induce a significant decrease in microvessel density in vivo, e.g. zoledronic acid inhibits proliferation and reduces the number and adhesion of circulating human endothelial cells [12]. Other hypotheses in the pathophysiology of $\mathrm{ONJ}$ in- criminate a direct soft-tissue toxicity of BPs, or an immune dysfunction pointed with the significant contribution of immunomodulators in the pathophysiology of the disease, in treatment with oral BPs and steroids [13].

\section{Rationale For Treatment With Bisphosphonates Or Deno- sumab:}

Diseases that affect bone can have debilitating effects on patients' lives by predisposing them to such events as fractures and other bone complications. These events can have a negative impact on morbidity, ability to work, and social activity [14].

\section{Osteoporosis:}

Efficacy of both low-dose oral bisphosphonate and low-dose denosumab treatments in reducing the fracture rate in osteoporosis has been clearly demonstrated in the clinical trial setting. In practice, however, the effectiveness of oral osteoporosis treatments is limited by poor levels of adherence by patients to their treatment regimen. This has been attributed to lack of understanding in patients about their condition and the associated fracture risk, as well as concerns about adverse events, such as MRONJ [15].

\section{Cancer Treatment Induced Bone Loss:}

Bone loss is a well-established risk associated with hormone ablation in prostate or breast cancer, although chemotherapy, radiotherapy, and TKIs may also play a role in dysregulating bone remodeling. Cancer treat-mentinduced bone loss is associated with an increased risk of fractures, which can be reduced with bisphosphonates or denosumab [16].

\section{Skeletal-Related Events In Patients With Malignancies In- Volving Bone:}

Bone is a common destination for metastases from primary solid tumors, particularly those originating in the breast or the prostate. Studies suggest that $68 \%$ of patients with prostate cancer and $73 \%$ of patients with breast cancer had bone metastases at postmortem examination; and that $95 \%$ to $100 \%$ of patients with multiple myeloma (MM) eventually develop bone lesions during the course of the disease. Bone metastases from solid tumors and bone lesions in MM frequently lead to skeletal-related events (SREs) (defined as pathologic fracture, radiation to bone, surgery to bone, and spinal cord compression) which place a considerable burden on patients and health care resources [17].

\section{Giant Cell Tumor Of Bone:}

Giant cell tumor of bone (GCTB) is a rare primary bone tumor with an incidence of approximately 0.1 to 1 per 1 million people per year, typically affecting young adults. In the majority of cases GCTB is benign, and metastasis is unusual. However, GCTB can be locally aggressive, and benign tumors may transform in to malignant high-grade sarcoma. Locally aggressive GCTB may require substantial surgical resection and impact significantly on bone stability. In clinical trials, high-dose denosumab treatment prevented tumor progression, induced primary tumor reduction, increased bone formation, and reduced pain in patients with GCTB. High-dose denosumab is approved for the treatment of adults and skeletally mature adolescents with GCTB [18].

\section{Paget Disease Of Bone:}

Paget disease of bone is a chronic metabolic bone disorder, which manifests as excessive and disorganized bone formation. Symptoms include pain, neurologic effects resulting from nerve compression, and hearing loss; furthermore, the disease may be associated with heart failure and hypercalcemia [19].

\section{Other Risks Associated With Bisphosphonates Or Deno- sumab Agents:}

The risk of some AEs other than MRONJ associated with the use of bisphosphonates and denosumab varies with the dose and duration of treatment. Bisphosphonates are nephrotoxic and are associated with upper gastrointestinal irritation (when used orally), acute-phase responses, and an increased risk of atrial fibrillation. More rarely, patients may be at increased risk of hypocalcemia and ocular inflammation. Denosumab is associated with AEs other than MRONJ, including hypocalcemia and musculoskeletal pain. The risk of developing hypocalcemia as a result of treatment with 
bisphosphonates or denosumab can be reduced by intake of sufficient dietary or supplemental calcium [20].

\section{MRONJ Risk Assessment:}

Low risk assessment.

Prevention: Before the start of the treatment.

No routine screening visit required.

Usual recommendations of preventive dental visits for the general population is applied.

If the patient has not complied with these recommendations, a check up should be advised.

\section{Prevention: during therapy}

Maintain optimal dental health.

Prophylactic Dental cleaning.

Tooth fillings.

Non traumatic treatments or prosthetics without bone anchoring.

Patient education on maintaining good oral hygiene.

\section{No special precautions apply}

Very low MRONJ risk.

All Dental procedures maybe performed as indicated.

Recommendations for the general population on preventive dental assessment apply.

\section{Elevated MRONJ Risk}

Prevention: before start of therapy.

Screening visit required.

Detect and treat pockets of occult infection.

Extract teeth with poor prognosis.

Check dentures.

Encourage smoking cessation.

Educate patient on recognising signs and symptoms of MRONJ.

Educate patients on maintaining good oral hygiene.

\section{Prevention During Treatment}

Invasive dental procedures require expert advice.

Including Dental extractions, periodontal surgery, root planing and implants.

Low threshold for referring patients to OMFS or a specialised dentist in case of unexplained symptoms.

Use prophylactic antibiotics in case of unavoidable or emergency procedures.

If MRONJ is suspected, consider starting $\mathrm{CHX}$ or empirical broad spectrum antibiotic treatment (Amoxicillin with clavilanic acid).

\section{Diagnosis Of MRONJ}

Key signs and symptoms.

It is important that dentists are confident in recognizing the signs and symptoms of MRONJ and are familiar with the staging system that has been established for this condition. Pain and signs of infection are the most frequent symptoms reported by patients, but MRONJ can be asymptomatic. Conditions commonly confused with MRONJ include alveolar osteitis. sinusitis, gingivitis and periodontitis, periapical pathology, odontalgia, atypical neuralgias, sarcoma, and chronic sclerosing osteomyelitis.

In most cases, a diagnosis of MRONJ requires the following

Current or previous treatment with bisphosphonates, denosum$\mathrm{ab}$, or antiangiogenic therapy.

An area of exposed bone, or bone that can be probed through an intraoral or extraoral fistula and has persisted for greater than 8 weeks.

No history of radiation therapy to the jaw or obvious metastatic disease of the jaw.

It should be noted that there are certain caveats to these general principles. An 8-week observation period may be appropriate in cases of nonhealing postextrac - tion sockets, but in many cases, the diagnosis is clear, and periods of observation are not necessary. Further - more, there are increasing reports of nonexposed forms of osteonecrosis, which should also be included in the differential diagnosis.

\section{Diagnostic Stages Of MRONJ}

The AAOMS has defined the stages of MRONJ to describe disease presentation and to facilitate the appropriate stratification of patients:

Stage 0-no clinical evidence of necrotic bone, but nonspecific clinical findings, radiographic changes, and symptoms.

Stage 1-exposed and necrotic bone/fistulae that can be probed to bone, asymptomatic, no evidence of infection.

Stage 2-exposed and necrotic bone/ fistulae that can be probed to bone, associated with infection.

Stage 3-exposed and necrotic bone/fistulae that can be probed to bone, associated with infection and additional complications. 


\section{Treatment Of MRONJ}

There is no defined treatment algorithm, but findings from a systematic review of treatment strategies for MRONJ suggested that stage-specific treatment approaches have a sound scientific foundation. The goal of MRONJ management should be control of infection, progression of bone necrosis, and pain. If MRONJ occurs while a patient is receiving high - dose bisphosphonate or denosumab, the need for continuation of treatment should be discussed with all involved, taking into account the severity and evolution of MRONJ, the oncologic disease burden and activity, and the wishes of the patient [21].

\section{Conservative Management}

Conservative management approaches include maintaining optimal oral hygiene, eliminating active dental and periodontal diseases, and application of topical antibacterial mouth rinses and systemic antibiotic therapy, as indicated by local guidelines. Such strategies may be used in cases where there is no obvious disease progression, uncontrolled pain, or discontinuation of bisphosphonate or denosumab therapy as a result of MRONJ.

\section{Surgical Management}

Recent evidence suggests that surgery is effective in reducing pain in patients with MRONJ and ultimately leads its resolution. Surgery is, therefore, indicated for patients with MRONJ whose disease does not respond to or is deemed unlikely to respond to conservative approaches. The following surgical principles have been proposed for the removal of necrotic bone in this patient group: "A full-thickness mucoperiosteal flap should be high and extended to reveal the entire area of exposed bone and beyond to disease-free margins; resection of the affected bone should be extended horizontally and inferiorly to reach healthy-appearing, bleeding bone; sharp edges should be smoothed; and primary soft tissue closure achieved" through appropriate mobilization and suturing to facilitate tension-free mucosal healing [22].

\section{Adjuvant Treatment Options}

In addition to the established conservative and surgical treatment options, several adjuvant treatments for MRONJ have been investigated, including laser-assisted surgical debridement/lowlevel laser therapy and the application of ozone oil or plateletrich plasma/platelet-derived growth factor to the surgical wound. However, it should be noted that these techniques have yielded conflicting results and have not yet been assessed in prospective controlled clinical trials.

\section{Developments In MRONJ Treatment}

MRONJ remains a topic for research and debate among the medical and dental communities. An improved understanding of how treatment with bisphosphonates or denosumab interacts with trigger events, such as oral infections or trauma, will help optimize the prevention and treatment of MRONJ.

The definition of MRONJ and, in particular, the diagnostic stages are subjects of ongoing debate. Specific and nonspecific radiographic features may be associated with clinical MRONJ, but im- aging criteria have yet to be included in the formal definition of the disease. Further clarification regarding the definition of stage $0 \mathrm{MRONJ}$ is required; its clinical relevance and benefit need to be clearly described in the MRONJ classification system. The precise definition of 'nonexposed' disease also needs to be established [23]. More research is needed on preclinical and clinical aspects of MRONJ, including further studies to confirm whether localized periodontal disease is an early form of MRONJ, and additional controlled trials should be conducted to establish the effectiveness of different treatment modalities. There is also an urgent need to define the characteristics of the increasingly reported cases of osteonecrosis of the jaw related to medications without known antiresorptive properties [24].

Patient-reported outcome data are needed, not only to help to establish which treatments are most effective but also to better understand the disease process. Although there is a higher risk of MRONJ in the oncology setting than in the osteoporosis setting, evidence suggests that the burden of disease may, paradoxically, be greater in the latter. Despite being a safety consideration associated with bisphosphonates and denosumab, MRONJ is not typically a focus of attention among patients, caregivers, or nurses, who rank it very low among factors that influence boneprotection treatment preferences [25].

\section{Conclusion}

MRONJ is a rare, but potentially serious, AE associated with different therapies (including chemotherapy) and specifically with high-dose/long-term use of bisphosphonates or denosumab. The development of MRONJ may compromise treatment, there by increasing the risk of pathologic fractures in those with osteoporosis and of fractures and other bone complications (SREs) in individuals with cancer. Minimizing the risk of MRONJ is critical, not only to prevent the pain and discomfort the disease can cause patients but also to maximize the benefit of treatment with bisphosphonates or denosumab. Dentists have a pivotal role to play in preventing MRONJ; through thorough assessment, prophylactic dental treatment, and close multi-professional teamwork, the risk of developing this condition can be reduced. To that end, it is important that dentists are able to identify patients at risk, are familiar with the required prophylactic treatment recommendations, and are aware of the diagnostic criteria and management strategies for MRONJ.

\section{References}

[1]. Khan AA, Morrison A, Hanley DA, Felsenberg D, McCauley LK, O'Ryan F, et al. Diagnosis and management of osteonecrosis of the jaw: a systematic review and international consensus. J Bone Miner Res. 2015 Jan;30(1):3-23.

[2]. Drake MT, Clarke BL, Khosla S. Bisphosphonates: mechanism of action and role in clinical practice. Mayo Clin Proc. 2008;83:1032-1045.

[3]. Luckman SP, Hughes DE, Coxon FP, Russell RG, Rogers MJ. Nitrogen-containing bisphosphonates inhibit the mevalonate pathway and prevent posttranslational prenylation of GTP-binding proteins, including Ras. J Bone Miner Res. 1998 Apr;13(4):581-9.

[4]. Gaudin E, Seidel L, Bacevic M, Rompen E, Lambert F. Occurrence and risk indicators of medication-related osteonecrosis of the jaw after dental extraction: a systematic review and meta-analysis. J Clin Periodontol. 2015 Oct;42(10):922-32.Pubmed PMID: 26362756.

[5]. Dhesy-Thind S, Fletcher GG, Blanchette PS, Clemons MJ, Dillmon MS, Frank ES, et al. Use of Adjuvant Bisphosphonates and Other Bone-Modifying Agents in Breast Cancer: A Cancer Care Ontario and American Society of Clinical Oncology Clinical Practice Guideline. J Clin Oncol. 2017 Jun 
20;35(18):2062-2081.Pubmed PMID: 28618241.

[6]. Ruggiero SL, Dodson TB, Fantasia J, Goodday R, Aghaloo T, Mehrotra B, et al. American Association of Oral and Maxillofacial Surgeons position paper on medication-related osteonecrosis of the jaw-2014 update. J Oral Maxillofac Surg. 2014 Oct 1;72(10):1938-56.

[7]. Barasch A, Cunha-Cruz J, Curro FA, Hujoel P, Sung AH, Vena D, et al. Risk factors for osteonecrosis of the jaws: a case-control study from the CONDOR dental PBRN. J Dent Res. 2011 Apr;90(4):439-44.

[8]. Thumbigere-Math V, Tu L, Huckabay S, Dudek AZ, Lunos S, Basi DL, et al. A retrospective study evaluating frequency and risk factors of osteonecrosis of the jaw in 576 cancer patients receiving intravenous bisphosphonates. Am J Clin Oncol. 2012 Aug;35(4):386-92.Pubmed PMID: 22561331.

[9]. Stopeck AT, Lipton A, Body JJ, Steger GG, Tonkin K, De Boer RH, et al. Denosumab compared with zoledronic acid for the treatment of bone metastases in patients with advanced breast cancer: a randomized, double-blind study. J Clin Oncol. 2010 Dec 10;28(35):5132-9.

[10]. Baron R, Ferrari S, Russell RG. Denosumab and bisphosphonates: different mechanisms of action and effects. Bone. 2011 Apr 1;48(4):677-92.

[11]. Marx RE, Sawatari Y, Fortin M, Broumand V. Bisphosphonate-induced exposed bone (osteonecrosis/osteopetrosis) of the jaws: risk factors, recognition, prevention, and treatment. J Oral Maxillofac Surg. 2005 Nov;63(11):156775.Pubmed PMID: 16243172.

[12]. McLeod NM, Brennan PA, Ruggiero SL. Bisphosphonate osteonecrosis of the jaw: a historical and contemporary review. surgeon. 2012 Feb $1 ; 10(1): 36-42$

[13]. Vescovi P, Merigo E, Meleti M, Manfredi M, Fornaini C, Nammour S, et al. Conservative surgical management of stage I bisphosphonate-related osteonecrosis of the jaw. Int J Dent. 2014 Feb 6;2014:107690.

[14]. Fischer S, Kapinos KA, Mulcahy A, Pinto L, Hayden O, Barron R. Estimating the long-term functional burden of osteoporosis-related fractures. Osteoporos Int. 2017 Oct;28(10):2843-51.

[15]. Eisenberg DF, Placzek H, Gu T, Krishna A, Tulsi BB. Cost and consequences of noncompliance to oral bisphosphonate treatment. J Manag Care Spec Pharm. 2015 Jan;21(1):56-65.Pubmed PMID: 25562773.
[16]. Ottanelli S. Prevention and treatment of bone fragility in cancer patient. Clin Cases Miner Bone Metab. 2015 May;12(2):116-129.

[17]. Coleman RE. Clinical features of metastatic bone disease and risk of skeletal morbidity. Clin Cancer Res. 2006 Oct 15;12(20):6243s-9s.

[18]. Rosen LS, Gordon D, Kaminski M, Howell A, Belch A, Mackey J, et al. Long-term efficacy and safety of zoledronic acid compared with pamidronate disodium in the treatment of skeletal complications in patients with advanced multiple myeloma or breast carcinoma: a randomized, double-blind, multicenter, comparative trial. Cancer. 2003 Oct 15;98(8):1735-44.Pubmed PMID: 14534891.

[19]. Wat WZ. Current perspectives on bisphosphonate treatment in Paget's disease of bone. Ther Clin Risk Manag. 2014;10:977-983.

[20]. Dalle Carbonare L, Zanatta M, Gasparetto A, Valenti MT. Safety and tolerability of zoledronic acid and other bisphospho- nates in osteoporosis management. Drug Healthc Patient Saf. 2010;2:121-137.

[21]. Hinson AM, Siegel ER, Stack BC Jr. Temporal correlation between bisphosphonate termination and symptom resolution in osteonecrosis of the jaw: a pooled case report analysis. J Oral Maxillofac Surg. 2015 Jan;73(1):53-62. Pubmed PMID: 25511956.

[22]. Ripamonti CI, Cislaghi E, Mariani L, Maniezzo M. Efficacy and safety of medical ozone $(\mathrm{O}(3))$ delivered in oil suspension applications for the treatment of osteonecrosis of the jaw in patients with bone metastases treated with bisphosphonates: Preliminary results of a phase I-II study. Oral Oncol. 2011 Mar;47(3):185-90.Pubmed PMID: 21310650.

[23]. Bedogni A, Fedele S, Bedogni G, Scoletta M, Favia G, Colella G, et al. Staging of osteonecrosis of the jaw requires computed tomography for accurate definition of the extent of bony disease. Br J Oral Maxillofac Surg. 2014 Sep;52(7):603-8.Pubmed PMID: 24856927.

[24]. Nicolatou-Galitis O, Kouri M, Papadopoulou E, et al. Osteonec- rosis of the jaw related to non-antiresorptive medications: a sys- tematic review. Support Care Cancer. 2018;26:S39-S364. eP035.

[25]. Hechmati G, Hauber AB, Arellano J, Mohamed AF, Qian Y, Gatta F, et al. Patients' preferences for bone metastases treatments in France, Germany and the United Kingdom. Support Care Cancer. 2015 Jan;23(1):21-8. 